\title{
Atomic Scale Tracking of a Charge Order Transition with Continuously Variable Temperature Cryo-STEM
}

Noah Schnitzer ${ }^{1}$, Elisabeth Bianco ${ }^{1}$, Alemayehu Admasu ${ }^{2}$, Jaewook Kim ${ }^{2}$, Sang-Wook Cheong ${ }^{2}$, Ismail El Baggari ${ }^{1}$ and Lena Kourkoutis ${ }^{1}$

${ }^{1}$ Cornell University, Ithaca, New York, United States, ${ }^{2}$ Rutgers University, Piscataway, New Jersey, United States

Strongly correlated materials feature rich phase diagrams with competing order parameters and anomalous emergent properties. In many systems, however, phenomena such as charge and magnetic order can only be accessed at low temperatures, necessitating studies under cryogenic conditions. Charge ordering, a spatial modulation in electron density observed in many strongly correlated systems, is associated with superconductivity, metal-insulator transitions, and colossal magnetoresistance [1-3]. Using high-angle annular dark field scanning transmission electron microscopy (HAADF-STEM), previous work has succeeded in mapping the periodic lattice distortions (PLDs) associated with charge ordering at atomic resolution in a manganite in both its low-temperature commensurate and high-temperature incommensurate states [4,5]. However, cryogenic HAADF-STEM has conventionally been performed at the base temperature of the cryogenic holder ( $~ 93 \mathrm{~K}$ for liquid nitrogen). Understanding the phase diagrams of these strongly correlated materials requires characterizing their structural and electronic properties at intermediate temperatures and across phase transitions. Here, we demonstrate atomic resolution HAADF imaging at intermediate cryogenic temperatures $(\sim 100-278 \mathrm{~K})$ in a charge ordered manganite, and track the PLD associated with charge ordering across a commensurate-incommensurate phase transition.

$\mathrm{Bi} 1-\mathrm{x}_{\mathrm{x}-\mathrm{y}} \mathrm{Ca}_{\mathrm{y}} \mathrm{MnO}_{3}(\mathrm{BSCMO})$ is a model charge ordered system with a $\mathrm{T}_{\mathrm{c}}$ of $\sim 300 \mathrm{~K}$ at doping $(\mathrm{x}=0.65$, $\mathrm{y}=0.48$ ), and a commensurate structure at liquid nitrogen temperature $\sim 93 \mathrm{~K}$. Above this temperature the material transitions to an incommensurate state [5]. Here, a continuously variable temperature cryogenic specimen holder (HennyZ) enables intermediate temperature cryo-STEM images across the commensurate-incommensurate phase transition (Fig. 1). Stable imaging conditions at temperatures between liquid nitrogen and room temperature are obtained by coupling liquid nitrogen rod cooling with local MEMS heating of the sample (Fig. 1d) [6]. HAADF STEM imaging was performed at $300 \mathrm{kV}$ on an aberration corrected FEI Titan Themis using a $21.4 \mathrm{mrad}$ convergence angle. Stacks of 20-50 images acquired with sub-microsecond pixel dwell times were registered and averaged to ensure adequate signal to handle drift and achieve a sufficient signal-to-noise ratio. Both A and B sites are clearly resolved across the temperature range, and the signal-to-noise ratio is sufficiently high to allow quantification of the picometer-scale PLD behavior. The PLD is readily identifiable in the Fast Fourier Transform (FFT) of the real space images (Fig. 2 a-c) as superlattice peaks with wavevectors near $1 / 3$ reciprocal lattice units (r.l.u.). The commensurate-incommensurate transition can be observed in the shift of the superlattice peaks with temperature (Fig. 2d). At low temperature the peaks are sharp and lie near the lattice-locked 1/3 r.l.u. position. With increasing temperature, the peaks begin to broaden and at $\sim 238 \mathrm{~K}$ shift inwards towards the Bragg peak to an incommensurate position. The capability shown here to track exotic electronic and structural phases across cryogenic temperatures will unlock a deeper understanding of phase transitions and the competition between order parameters present at these temperatures. [7] 

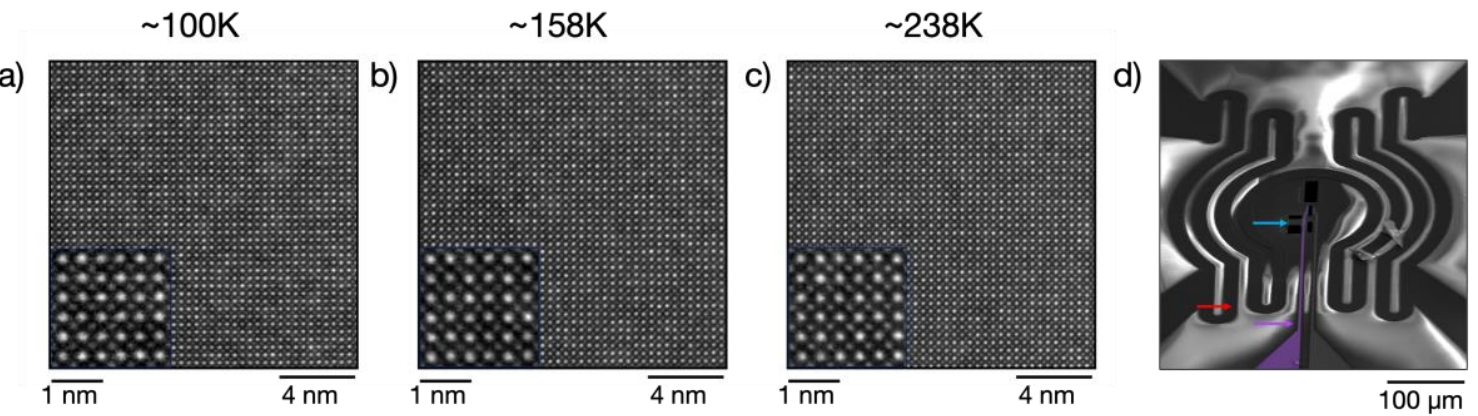

Figure 1. $16 \mathrm{~nm}$ field of view atomic-resolution HAADF-STEM images of BSCMO imaged along the [100] axis at (a) $\sim 100 \mathrm{~K}$, (b) $\sim 158 \mathrm{~K}$, (c) $\sim 238 \mathrm{~K}$. (a-c insets) Both A and B sites are clearly resolved at these intermediate cryogenic temperatures. (d) SEM image of a FIB lamellae mounted on a MEMS insitu heating chip. Lamellae (blue arrow), heating coil (red arrow), and electrical contact (here unused, purple arrow and overlay) indicated.

a)

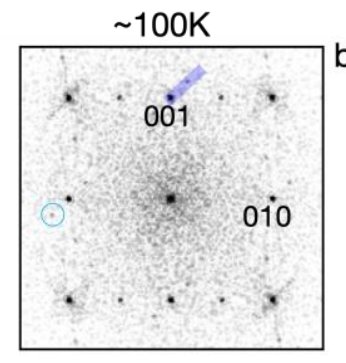

b)

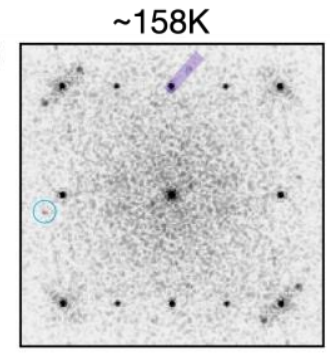

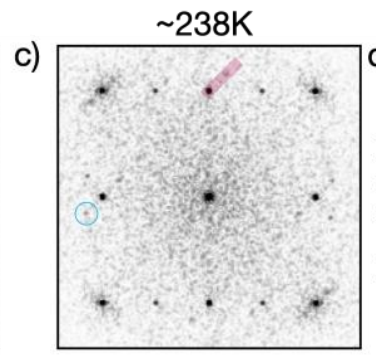

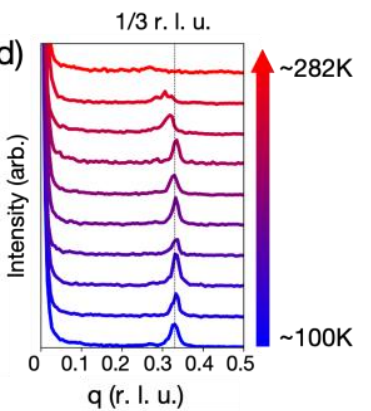

Figure 2. FFTs (Hough filtered, log transformed) of HAADF-STEM images at (a) 100 K, (b) 158 K, (c) $\sim 238$ K. Superlattice peaks from the PLD (e.g. blue circle) decorate each Bragg peak. (d) Line profiles across the superlattice peaks in the FFT as a function of temperature. With increasing temperature, the wavevector position decreases and becomes incommensurate. Representative profiles indicated by colored lines in $(a, b, c)$. Plotted data in (d) is average of profiles of every 1st and 2nd order Bragg peak from 106 $\mathrm{nm}$ field of view STEM images.

\section{References}

[1] J. Chang, et al. Nature Physics 8, 871-876 (2012).

[2] J. A. Wilson, F. J. Di Salvo, S. Mahajan. Advances in Physics 24, 117-201 (1975).

[3] G. C. Milward, M. J. Calderón, P. B. Littlewood. Nature 433, 607-610 (2005).

[4] B. H. Savitzky, et al. Nature Communications 8, 1833 (2017).

[5] I. El Baggari, et al. PNAS 115, 1445-1450 (2018).

[6] B. H. Goodge, et al. arXiv:2001.11581

[7] This work was supported by AFOSR (FA 9550-16-1-0305) and NSF (DMR-1539918, DMR-1429155, DMR-1719875). 